\title{
Lumbar Puncture in the Presence of Raised Intracranial Pressure
}

\author{
G. P. DUFFY,* M.B., F.R.C.S., M.R.C.P.ED.
}

Cummary : Study of 30 patients with raised intracranial pressure whose condition worsened after lumbar puncture emphasizes the danger of carrying this out in the presence of raised intracranial pressure. In half the cases deterioration was immediate and dramatic, and in the other half it occurred within 12 hours. Probably a tentorial or cerebellar pressure cone, or both, had formed before lumbar puncture, and the procedure made this worse and caused the clinical deterioration.

A history of progressive headache associated with mental changes, and the development and progression of localizing neurological signs were the two features suggestive of varied intracranial pressure found most constantly in this series. A good quality plain $x$-ray film is important in the diagnosis of this condition.

\section{Introduction}

The effects of distortion and compression of the midbrain and medulla, and disturbance of their blood supply as a result of herniation of the unci of the temporal lobes through the tentorial hiatus or of the cerebellar tonsils through the foramen magnum, have been clearly and repeatedly described (Collier, 1904 ; Meyer, 1920 ; Jefferson, 1938 ; Finney and Earl Walker, 1962). That these effects can be precipitated by interfering with the intracranial dynamics in the presence of raised intracranial pressure, particularly if there is already distortion of the intracranial contents, has been pointed out by Jefferson (1938), Smyth and Henderson (1938), and Finney and Earl Walker (1962). For this reason it is usual in British medical practice to teach against the performance of lumbar puncture in the presence of raised intracranial pressure unless the information to be obtained is vital to treatment, and then only if surgical facilities for dealing with any ensuing ill-effects are immediately available. Despite this there are many who believe that the dangers of this procedure have been exaggerated (Masson, 1929 ; Norlén and Wickbom, 1958 ; Korein, Cravioto, and Leicach, 1959).

Junior residents often seem to be unaware of the danger, and it is common practice in some hospitals for the cerebrospinal fluid to be examined in any case in which there is a suspicion of an intracranial lesion. As a result midbrain and medullary compression syndromes following lumbar puncture are not uncommon causes for the emergency admission of patients to a neurosurgical department. This can be illustrated by the fact that in the 12 months January to December 196630 such cases were admitted-27 to the Midland Centre for Neurosurgery and Neurology and three to the Department of Neurosurgery, Queen Elizabeth Hospital, Birmingham. All of these cases were investigated with angiography or ventriculography after admission to the neurosurgical centre, and 25 of them had subsequent surgery. These cases form the material for the present study, which is aimed at showing not only the danger of lumbar puncture in the presence of raised intracranial pressure but also the clinical pictures which should serve as a warning to contraindicate it, the results of ill-advised

* Consultant Neurosurgeon to the Midland Centre for Neurosurgery and
Neurology, Smethwick, and the West Bromwich, Coventry, and Neurology, Smethwick, and the West Bromwich, Coventry, and
South Warwickshire Groups, Birmingham Regional Hospital Board. cerebrospinal fluid examination, and the types of pathological process responsible.

\section{Clinical Features Before Lumbar Puncture}

Headache.-All but one of the cases studied gave headache as a prominent symptom, of ten preceding other symptoms and frequently increasing in severity.

History of Progressive Localizing Symptoms.- Seventeen $(57 \%)$ cases showed a history of a progressive development of localizing symptoms. These symptoms were progressive dysphasia, progressive hemiparesis, or progressive ataxia.

Presence of Localizing Signs on Initial Examination.Twenty-two $(73 \%)$ cases showed localizing signs on examination before the lumbar puncture was performed. These included dysphasia, hemiparesis, hemianopia, lateralizing reflex inequalities, nystagmus and lateralizing ataxia, and pupillary inequalities.

History of Progressive Mental Changes.-Fourteen (47\%) cases had a history of progressive and definite alteration in personality, and seven ( $23 \%$ ) further cases had developed increasing drowsiness before lumbar puncture.

Papilloedema.-Nine $(30 \%)$ cases were recognized to have papilloedema before the lumbar puncture was performed in the referring hospital. On initial examination in the neurosurgical centre $20(67 \%)$ cases were thought to show definite papilloedema. In nearly all the cases less than 48 hours had elapsed between the two fundal examinations.

Meningitic Symptoms.-Five cases presented with a previous history of sinus or ear infection and signs suggestive of a meningitis. Initial lumbar puncture produced a cerebrospinal fluid with only a moderately raised cell count, but the cerebrospinal fluid was found to be sterile. Treatment for meningitis was started and repeated lumbar punctures were performed. Deterioration followed the second or later lumbar puncture. Subsequent events showed a cerebral abscess to be present.

$X$-ray Changes.-Plain skull $x$-ray films showed lateral displacement of a calcified pineal gland in $25 \%$ of those cases with supratentorial lesions. Definite erosion of the posterior clinoid processes suggestive of chronically raised intracranial pressure was present in $30 \%$ of these $x$-ray films.

Of the 30 cases 27 showed several if not all of the above features before lumbar puncture was performed.

\section{Changes After Lumbar Punctures}

Immediate Loss of Consciousness (Table I).-Three of these patients stopped breathing during the lumbar puncture or immediately after withdrawal of the lumbar puncture needle. Seven rapidly developed unequal pupils.

TABle I.-Changes After Lumbar Puncture

Immediate loss of consciousness Rapid change in conscious leve

Rapid change in signs without alteration in conscious level

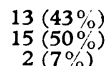

Rapid Change in Conscious Level.-The change in conscious level in all 15 cases came on within 12 hours of lumbar puncture, and was followed by progressive deterioration and 
worsening neurological signs. Three of them developed unequal pupils.

Rapid Change in Signs without Alteration in Conscious Level.-In one case one pupil immediately became enlarged and non-reacting, and in the other case a dilated right pupil rapidly developed, this being associated with the development of a left hemiparesis and a left hemianopia but no alteration in conscious level.

\section{Diagnosis}

Table II shows the pathological processes present and their distribution between the supratentorial and subtentorial compartments. It will be noted that half these cases had nonmalignant, potentially curable lesions.

\begin{tabular}{|c|c|c|c|c|}
\hline Supratentorial, $23\left(77^{\circ}{ }_{0}\right)$ & & Subtentorial, & $(23$ & \\
\hline Glioma $\ldots \quad \ldots \quad \ldots \quad \ldots$ & 10 & Glioma . & . & .. \\
\hline Abscesses treated as meningitis & & Haemangioblastoma & . & \\
\hline with more than one L.P. . . & 5 & Acoustic neuroma & . & .. \\
\hline Abscess $\quad \ldots \quad \ldots$ & 3 & Abscess .. .. & . & .. \\
\hline Meningioma $\ldots$ & 3 & & & \\
\hline Secondary melanoma $\quad \ldots$ & 1 & & & \\
\hline Massive hemisphere infarction & 1 & & & \\
\hline
\end{tabular}

Cerebral Abscesses Investigated with Repeated Lumbar Punctures and Treated as Meningitis.-A rather special category of case was found in which abscesses were treated as meningitis and investigated with repeated lumbar punctures, and as these form an important group a brief outline is given of their clinical features.

Case 4.-Left frontal sinusitis treated with antibiotics one month previously. Retro-orbital headache and vomiting associated with pyrexia and meningism led to lumbar puncture and initiation of treatment for meningitis. Three lumbar punctures performed over a period of 10 days, and before the third lumbar puncture a minimal left hemiparesis had developed. Patient became unconscious during the third lumbar puncture, but recovered to drowsiness and left hemiplegia.

Case 9.-Meningitic symptoms following sinusitis one month previously. Lumbar puncture performed at that time and treatment initiated for a few days with relief of symptoms. Three days before present admission, headache, fever, and meningism had recurred. Second and third lumbar punctures were performed within 24 hours of each other, the last followed by rapid deterioration to unconsciousness.

Case 12.-Chronic discharging otitis media. A one-month history of headache and vomiting associated with meningism. Treatment for meningitis initiated after first lumbar puncture. Second lumbar puncture was performed 12 days later, and the patient became decerebrate with dilated non-reacting pupils.

Case 23-Eight-week history of recurrent right otitis media, sixweek history of headaches, vomiting, and pyrexia persisting despite antibiotic treatment. Meningism led to the first lumbar puncture. After the second lumbar puncture, 10 days later, a marke 1 increase in drowsiness occurred, associated with enlargement of the right pupil.

Case 24.-Frontal sinusitis treated with antibiotics for two weeks. Two weeks before admission patient developed meningism and after first lumbar puncture was treated for meningitis. Lumbar-punctured on three occasions over 12 days. After third lumbar puncture a marked increase in drowsiness occurred associated with the development of a left hemiparesis.

In every case the cerebrospinal fluid obtained at initial and subsequent lumbar puncture was sterile. In none was the sugar concentration in the cerebrospinal fluid more than marginally reduced, and the total white cell count varied between 20 and 6,000 cells per cu. mm. All these cases were shown by investigation, operation, or necropsy to have localized supratentorial abscesses without evidence of meningitis.

$$
\text { TABLE III.-Outcome }
$$

Died within 10 days of

Died within 6 months of L.P.

(1)

Improved but severely disabled

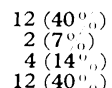

Outcome (Table III).- Of the 12 patients who died within 10 days of lumbar puncture eight had been precipitated into immediate coma following the lumbar puncture.

\section{Discussion}

Though one cannot be certain which of the signs and symptoms present before lumbar puncture were directly due to the primary lesion by virtue of its site and which were due to dislocation of the intracranial contents by the size of the lesion and the high intracranial pressure, both factors probably played a part. It is also probable that in all cases a tentorial or a cerebellar pressure cone, or both, had already formed before the lumbar puncture was carried out, and deterioration after the procedure was due to a worsening cone effect. Rapid progression of the primary disease might have caused a similar deteriora tion, but it is too much of a coincidence that this should have happened in each patient at precisely that time, and care was taken to include in the series only cases having a very definite and closely time-related deterioration after lumbar puncture. In half the cases this was immediate and dramatic; in others there was a delay, but in each case it occurred within 12 hours, and once the deterioration had started it became progressive and increasingly rapid. It is thought by some safe to lumbarpuncture in the presence of raised intracranial pressure if only a small amount of fluid is withdrawn. This does not allow for the seepage of the cerebrospinal fluid through the puncture hole in the theca after the needle is withdrawn. This seepage may be considerable and a partial explanation of a continuing and worsening herniation.

The clinical assessment of the presence of raised intracranial pressure is dependent on the consideration of several features. Those most constantly present in this series were the history of progressive headache associated with progressive mental changes and the development and progression of localizing neurological signs. Papilloedema was not a constant finding even when the history suggested that raised intracranial pressure had been present for some time. The value of observing the displacement of a calcified pineal gland on plain $x$-ray film as an indication of the presence of an intracranial space-occupying lesion, and the importance of recognizing erosion of the posterior clinoid processes, have been described in detail (Lilja 1948 ; Nordmark, 1949 ; Young, 1949 ; Du Boulay, 1965). Nearly half of the cases in this series showed such $x$-ray changes. Plain $x$-ray films should always be taken before lumbar puncture if raised intracranial pressure is suspected, but they must be straight and of good quality.

If the five cerebral abscesses investigated with repeated lumbar punctures are excluded, only three cases in this series did not show at least two different features suggesting raised intracranial pressure. One of these cases was a cerebral abscess which did show a pineal shift on straight $x$-ray examination. The second was a massive hemisphere infarction following a carotid artery occlusion, and the third was a secondary melanoma into which haemorrhage had occurred. Those cases where papilloedema was not recognized before lumbar puncture all showed other convincing features of an intracranial spaceoccupying lesion. Half of the cases in this series showed levels of cerebrospinal fluid pressure, as measured at lumbar puncture, within the limits of normal despite raised intracranial pressure later being proved. In the presence of distortion of the intracranial contents and partial obstruction of the flow of cerebrospinal fluid, manometric measurements of the lumbar cerebrospinal fluid pressure may not give a reliable index of the level of intracranial pressure.

The cases of cerebral abscess presenting in a meningitic manner and investigated with repeated lumbar punctures are classifiable as a syndrome which when recognized should contraindicate further lumbar puncture and initiate a request for specialist investigation. This syndrome consists of a history 
of an inflammatory process involving the ear, nose, or sinuses, followed after a period by meningitic signs and symptoms. Initial lumbar puncture produces a fluid containing inflammatory cells but with a doubtfully low sugar content and in which and from which no organisms can be seen or cultured. In these cases the possibility of the presence of an intracranial abscess should be carefully considered and investigated but not by further lumbar puncture. The conversion of a patient with an eminently treatable cerebral abscess into one with secondary irreversible brain-stem damage from tentorial pressure cone formation is an iatrogenic tragedy that should be avoidable.

I am grateful to Dr. E. R. Bickerstaff, Mr. J. G. Hamilton, Mr. J. M. Small, and Mr. E. A. Turner for allowing me to study those cases admitted under their care.

\section{REFERENCES}

Collier, J. (1904). Brain, 27, 490

Du Boulay, G. H. (1965). Principles of X-ray Diagnosis of the Skull. London.

Finney, L. A., and Earl Walker, A. (1962). Transtentorial Herniation. Springfield, Illinois.

Jefferson, G. (1938). Arch. Neurol. Psychiat. (Chic.), 40, 857. Korein, J., Cravioto, H., and Leicach, M. (1959). Neurology (Minneap.),
9, 290 .

Lilia, B. (1948). Acta radiol. (Stockh.), 30, 129.

Masson. C. B. (1929). Arch. Neurol. Psychiat. (Chic.), 21, 1141.

Meyer, A. (1920). Arch. Neurol. Psychiat. (Chic.), 4, 387.

Nordmark, B. (1949). Acta radiol. (Stockh.), 32, 461.

Norlén, G., and Wickbom, I. (1958). 7. Neurol. Neurosurg. Psychiat., 21, 1 .

Smyth, G. E., and Henderson, W. R. (1938). F. Neurol. Psychiat., 1,

Young, B. R. (1949). Radiology, 53, 625.

\title{
Width of Clavicular Cortex in Osteoporosis
}

\author{
H. C. ANTON,* M.B., ch.B., F.F.R.
}

Brit. med. F., 1969, 1, 409-411

\begin{abstract}
Summary : Thinning of the upper cortex of the clavicle, $\checkmark$ measured on a standard chest radiograph, may help in the diagnosis of osteoporosis. No precise level at which osteoporosis occurs can be given, but a reading of $1.5 \mathrm{~mm}$. or under is indicative of osteoporosis, while a smaller incidence is associated with readings of $2 \mathrm{~mm}$. and above. There is a significant correlation between thinning of the clavicular cortex and other radiological indications of osteoporosis. Thinning may occasionally point to unsuspected bone disease, in osteomalacia as well as in osteoporosis. As chest radiographs are taken in a high proportion of both outpatients and inpatients, the method has a wide applicability.
\end{abstract}

\section{Introduction}

Many excellent radiological methods for assessment of osteoporosis have been developed, such as the osteoporosis index of Barnett and Nordin (1960) and the ulnar densitometric method (Doyle, 1961). Such methods are rather time-consuming, and are generally used only in individuals or in groups already suspected of osteoporosis, or thought to be liable to it. Measurement of the cortical thickness of the radius, described by Meema (1963), is a valuable though simple method, but again radiographs of the forearm are not likely to be available save by special request. It seemed that there was a place for a simple method which might help in the detection of osteoporosis from a widely employed radiograph as well as in the assessment of its severity. This paper reports the value of estimations of the width of the cortex of the clavicle from routine chest films.

\section{Methods}

The thickness of the upper cortex of either clavicle (henceforward termed the C.C.T.) has been measured, on a standard posteroanterior chest radiograph, to the nearest $0.5 \mathrm{~mm}$. using an ordinary rule. The midpoint of the clavicle is taken for the reading, as the cortex thins appreciably towards the medial aspect of the clavicle and may show a double cortex at its lateral aspect. Furthermore, in the outer half of the cortex there is occasionally a localized cortical thickening associated with a nutrient foramen. The lower cortex proves unsuitable for measurement, as it is often poorly defined and sometimes, though rarely, is absent when other bones show normal cortices. It was decided not to compare the C.C.T. with the total width of the clavicle, as it seemed unlikely that this ratio would show any advantage, and because it would detract from the simplicity of the method.

The investigation is in two main parts. In the first part an attempt was made to find if there were differences in the C.C.T. related to the age and sex of the patient. For this purpose measurements of the C.C.T. were made on 120 randomly selected chest radiographs from both inpatients and outpatients during routine reporting in a general hospital. The readings were made before the age of the patient was noted. As a considerable proportion of the patients were elderly it is inevitable that some cases of osteoporosis will be included in the series. The results are given in Table I.

As the first part of the study suggested that a C.C.T. of $1.5 \mathrm{~mm}$. or under might be associated with osteoporosis, in that none of those below 40 years old showed such a figure, a record of consecutive cases with such a C.C.T. -56 in all, including the 15 relevant cases from the first part of the study -was kept over a period of several months. Their radiographs and case records were studied to determine whether a low C.C.T. was associated with other evidence of osteoporosis. This forms the basis of the second part of the study, the findings being presented in Table II.

Of the 56 cases with a C.C.T. of $1.5 \mathrm{~mm}$. or under, 25 were excluded because no radiographs other than chest ones were available. Five further cases were excluded from Table II, as only spine films were available, and these showed no evidence of wedging or biconcavity of the vertebral bodies. They were, however, included as negative cases in Table III. The work of Doyle, Gutteridge, Joplin, and Russell Fraser (1967) indicates that the subjective assessment of spinal osteoporosis is apt to 